\title{
Environmental contamination related to SARS-CoV-2 in ICU patients
}

\author{
Alexandra Lomont ${ }^{1,2,3}$, Marouane Boubaya ${ }^{4}$, Warda Khamis ${ }^{2,5}$, \\ Antoine Deslandes ${ }^{1}$, Hugues Cordel ${ }^{6}$, Delphine Seytre ${ }^{1}$, Chakib Alloui ${ }^{7}$, \\ Célie Malaure $^{7}$, Nicolas Bonnet ${ }^{2,8}$, Etienne Carbonnelle ${ }^{2,3,7}$, Yves Cohen ${ }^{2,8,9}$, \\ Hilario Nunes ${ }^{2,5,10}$, Olivier Bouchaud ${ }^{6,11}$, Jean-Ralph Zahar ${ }^{1,2,3}$ and \\ Yacine Tandjaoui-Lambiotte ${ }^{8,10}$ on behalf the AIRMIC study group
}

Affiliations: ${ }^{1}$ Clinical Microbiology Dept and Infection Control Unit, Hôpitaux Universitaires Paris-Seine-SaintDenis, Bobigny, France. ${ }^{2}$ University Sorbonne Paris Nord, Bobigny, France. ${ }^{3}$ INSERM UMR 1137, InfectionAntimicrobials-Modeling-Evolution, Paris, France. ${ }^{4}$ Clinical Research Unit, Hôpitaux Universitaires ParisSeine-Saint-Denis, Bobigny, France. ${ }^{5}$ Pulmonology Dept, Hôpitaux Universitaires Paris-Seine-Saint-Denis, Bobigny, France. 'Infectious and Tropical Diseases Dept, Hôpitaux Universitaires Paris-Seine-Saint-Denis, Bobigny, France. ${ }^{7}$ Clinical Microbiology Dept, Hôpitaux Universitaires Paris-Seine-Saint-Denis, Bobigny, France. ${ }^{8}$ Intensive Care Unit, Hôpitaux Universitaires Paris-Seine-Saint-Denis, Bobigny, France. ${ }^{9}$ INSERM U942, BIOmarkers in CArdioNeuroVAScular diseases (BIOCAVAS), Paris, France. ${ }^{10}$ INSERM U1272, Hypoxia \& Lung, Bobigny, France. ${ }^{11}$ Education and Health Practices Laboratory EA 3412, University Sorbonne Paris Nord, Bobigny, France.

Correspondence: Alexandra Lomont, Infection Control Unit, Clinical Microbiology Department, GH Paris Seine Saint-Denis, AP-HP, Bobigny, France. E-mail: alexandra.lomontđaphp.fr

\section{ABSTRACT}

Background: The coronavirus disease 2019 (COVID-19) outbreak is a primary global concern, and data are lacking concerning risk of novel severe acute respiratory syndrome coronavirus 2 (SARS-CoV-2) environmental contamination.

Objective: To identify risk factors for SARS-CoV-2 environmental contamination in COVID-19 patients admitted to the intensive care unit (ICU).

Methods: A prospective single centre 1-day study was carried out in an ICU. Four surfaces (the ventilator control screen, the control buttons of the syringe pump, the bed rails and the computer table located $>1 \mathrm{~m}$ away from the patient) were systematically swabbed at least $8 \mathrm{~h}$ after any cleaning process. We analysed clinical, microbiological and radiological data to identify risk factors for SARS-CoV-2 environmental contamination.

Results: $40 \%$ of ICU patients were found to contaminate their environment. No particular trend emerged regarding the type of surface contaminated. Modality of oxygen support (high-flow nasal cannula oxygenation, invasive mechanical ventilation, standard oxygen mask) was not associated with the risk of environmental contamination. Univariate analysis showed that lymphopenia $<0.7 \times 10^{9} \cdot \mathrm{L}^{-1}$ was associated with environmental contamination.

Conclusion: Despite small sample size, our study generated surprising results. Modality of oxygen support is not associated with risk of environmental contamination. Further studies are needed.

@ERSpublications

Environmental contamination due to \#SARSCoV2 occurs in $40 \%$ of ICU patient rooms. No difference is observed between different modalities of oxygen support (high-flow nasal cannula oxygenation, invasive mechanical ventilation, standard oxygen mask). https://bit.ly/3kgxTKx

Cite this article as: Lomont A, Boubaya M, Khamis W, et al. Environmental contamination related to SARS-CoV-2 in ICU patients. ERJ Open Res 2020; 6: 00595-2020 [https://doi.org/10.1183/ 23120541.00595-2020]. 


\section{Introduction}

The current coronavirus disease 2019 (COVID-19) pandemic has raised several concerns and questions. One of the major issues lies in our incomplete knowledge of the mode of transmission of the severe acute respiratory syndrome coronavirus 2 (SARS-CoV-2) responsible for the disease COVID-19 [1, 2].

Recent studies carried out regarding the risk of environmental contamination have shown conflicting results. Some authors suggested low or no environmental [3] contamination while others suggested a higher risk [4]. These discrepancies can be largely explained by many confounding factors, some related to patients' profiles (such as symptom severity) [5], others related to the method of investigation (such as delay between the onset of symptoms and the collection of samples).

Patients admitted to intensive care units (ICU) with severe respiratory involvement of COVID-19 could have higher levels of virus and therefore be responsible for more environmental contamination. The respiratory support required by ICU patients could increase environmental contamination because of aerosol-generating procedures (AGP) such as oxygen therapy, noninvasive ventilation (NIV), high-flow nasal cannula oxygenation (HFNO) and invasive mechanical ventilation (IMV) after endotracheal intubation. These therapies are thought to generate droplets or aerosols $[6,7]$ and could increase the risk of environmental contamination and transmission to healthcare workers [7, 8]. A recent publication put the case for a low risk of bio-aerosol dispersion due to HFNO use in SARS-CoV-2 patients [9] leading intensivists to have more questions than answers about SARS-CoV-2 environmental contamination in COVID-19 patients admitted to the ICU. Avoiding infection of healthcare workers is a major challenge in preventing healthcare system collapse in a worldwide health crisis. As personal protective equipment (PPE) supply has been a problem for ICU staff worldwide, improving our knowledge of environmental contamination in the ICU should be a priority.

The aim of this study was to assess the burden of SARS-CoV-2 environmental contamination and to evaluate the risk factors associated with an ICU population.

\section{Methods}

This prospective single-centre 1-day study was conducted in the ICU of Avicenne Hospital, a French 500 -bed teaching hospital. All patients admitted to the ICU with a recent $(<48 \mathrm{~h})$ SARS-CoV-2 positive result by real-time PCR were included.

All patients were confined to the ICU ward, which was a single room at neutral room air pressure, with the windows open and door closed. No visitors were allowed except for patients at the end of life. Staff continuously wore a surgical mask, glasses (or visor) and surgical gown. An FFP2 mask was used in cases of AGP and gloves in cases where there was exposure to body fluid.

Four critical (i.e. frequently touched) surfaces were systematically swabbed at least $8 \mathrm{~h}$ after any cleaning process: the ventilator control screen, the control buttons of the syringe pump, bed rails and the computer table located $>1 \mathrm{~m}$ away from the patient. For each patient demographic and medical history were recorded, focusing on data expected to modify viral excretion: immunocompromised status and lymphopenia $\left(<0.7 \times 10^{9} \cdot \mathrm{L}^{-1}\right)$, smoking status, presence of fever, diarrhoea or cough, and angiotensin-converting enzyme (ACE) inhibitor use.

Since several molecules were thought to influence viral load, data on their use were collected. In our hospital during the study period (March to April 2020), hydroxychloroquine was administered as a standard of care to all patients except where there was a contraindication. Intensivists stopped using lopinavir in the early days of the epidemic because of the high frequency of side effects due to drug interactions; therefore, none of the patients included in our study was treated with lopinavir. Remdesivir was not available in our hospital at the time of the study. At the time of the data collection, safety of steroid use in ICU patients infected with SARS-CoV-2 was unknown. Therefore the standard of care in our hospital was not to use steroids if SARS-CoV-2 was positive, which was the case of all patients included.

Type of respiratory support (standard oxygen mask with spontaneous ventilation, HFNO, NIV and IMV) and delays thought to impact the environmental contamination (delay between first symptoms and sampling, delay between hospital admission and sampling, delay between room admission and sampling) were recorded. Risk factors of severe respiratory involvement were recorded (body mass index, hypertension, extended lung involvement on thoracic high-resolution computed tomography (HRCT)) Patients' viral load was estimated using quantification of cycle threshold $\left(\mathrm{C}_{\mathrm{T}}\right)$.

A trained member of the infection control team performed the environmental sampling. A $10-\mathrm{cm}^{2}$ surface was systematically sampled using a pre-moistened nylon-tipped flocked swab (Copan Diagnostics, Murrieta, CA, USA). Swabs were rotated to ensure full contact of all parts of the swab tip and the surface. 
Real-time PCR to detect SARS-CoV-2 was performed on all samples, using the same protocol as our routine diagnosis of SARS-CoV-2 infections in the pandemic context. Nucleic acid extraction was performed using an RSC blood DNA kit on a Maxwell ${ }^{\circ}$ RSC device (Promega, Charbonnières-les-Bains, France), according to the manufacturer's recommendations, with an input sample volume of $300 \mu \mathrm{L}$ and an elution volume of $60 \mu \mathrm{L}$. Real-time PCR was performed strictly according to the Charité-Berlin protocol [10] targeting the $\mathrm{E}$ gene, coding for envelope protein, pangenomic of SARS-CoV-1 and SARS-CoV-2, on a QuantStudio7 (Thermo Fisher, Les Ulis, France) device.

\section{Statistical analysis}

Categorical variables were described as frequencies and percentages. Quantitative variables were described as the median (interquartile range). The incidence of environmental contamination was estimated with a $95 \%$ confidence interval.

The relationship between contaminated and non-contaminated rooms and potential predictors was investigated by a Chi-squared test or Fisher's exact test for categorical variables and Mann-Whitney test for quantitative variables. Due to the small sample size, no multivariate analysis was performed. All tests were two-sided at the 0.05 significance level. Statistical analysis was performed using the R 3.5.2 statistical package (R Foundation for Statistical Computing, Vienna, Austria: www.R-project.org).

\section{Results}

Between 26 March and 20 April 2020, 117 environmental samples were collected from 30 patients. Twenty-eight series were completed with four samples performed for each. One series was missing one sample (ventilator control screen) and another series was missing two samples (ventilator control screen and computer table).

Demographic and medical data are reported in table 1. Patients' median age was 61 years (range 21-81 years) and there were 21 men and nine women. The median delay between first symptoms and sampling was 11.5 days. Cough and diarrhoea within $24 \mathrm{~h}$ from sampling were observed in 16 (55\%) and 2 (7\%) patients, respectively. Median temperature was $38^{\circ} \mathrm{C}$ (range $36.5-40.2^{\circ} \mathrm{C}$ ). All 30 patients were nonsmokers. Four patients were treated with immunosuppressive drugs, two of them for a low-grade lymphoma, one for anti-neutrophil cytoplasmic autoantibody vasculitis and one for renal transplant.

Twelve patients (40\%) had one or more environmental samples testing positive for SARS-CoV-2. Virus RNA was amplified in 29 out of 117 samples (i.e., positives). Eight (29\%), seven (23\%), seven (23\%) and seven $(24 \%)$ of the samples for ventilator control screen, syringe pump control button, bed rails and computer table tested positive, respectively (see table 2). These results show that less than a quarter of the samples collected were positive for the RNA virus on the different surfaces tested. Among those surfaces no particular trend emerged in terms of location for positive samples. Of note, all $\mathrm{C}_{\mathrm{T}}$ values were high (median (min-max): 36 (29-39)) reflecting a low viral load in samples.

On univariate analysis, the respiratory symptoms (respiratory rate, cough), the extent of radiological damage on thoracic HRCT and the $\mathrm{C}_{\mathrm{T}}$ for patients' real-time PCR were not associated with risk of environmental contamination. Modality of oxygen support (HFNO, IMV, standard oxygen mask, NIV) was not associated with risk of environmental contamination. Among the nine patients chronically treated with ACE inhibitors, only one had SARS-CoV-2 environmental contamination ( $\mathrm{p}=0.05)$. Two immunocompromised patients had environmental samples positive for SARS-CoV-2, and lymphopenia $\left(<0.7 \times 10^{9} \cdot \mathrm{L}^{-1}\right)$ was associated with a higher environmental contamination $(\mathrm{p}=0.02)$. Neither delay between first symptoms and sampling nor fever was associated with environmental contamination.

\section{Discussion}

In this prospective cohort study conducted in ICU patients we identified environmental contamination in $40 \%$. In line with several other studies [11, 12], our results confirm the existence of SARS-CoV-2 environmental contamination in COVID-19 patients admitted to the ICU for acute respiratory failure. One of the important findings was the absence of significant difference in the frequency of positive results when comparing the different sites sampled. It is important to point out that the computer table located $>1 \mathrm{~m}$ away from the patient was as contaminated as the other surfaces. Because the computer table was the farthest away surface in our selection, we expected contamination to be the lowest. As our patients did not have access to the computer table, it is possible that contamination was airborne or from healthcare workers' hands. Guo et al. [11] reported similar results for the computer mouse, but there are no data for the distance between the computer mouse and the patients or modality of use of the computer (dedicated to healthcare workers or not). 


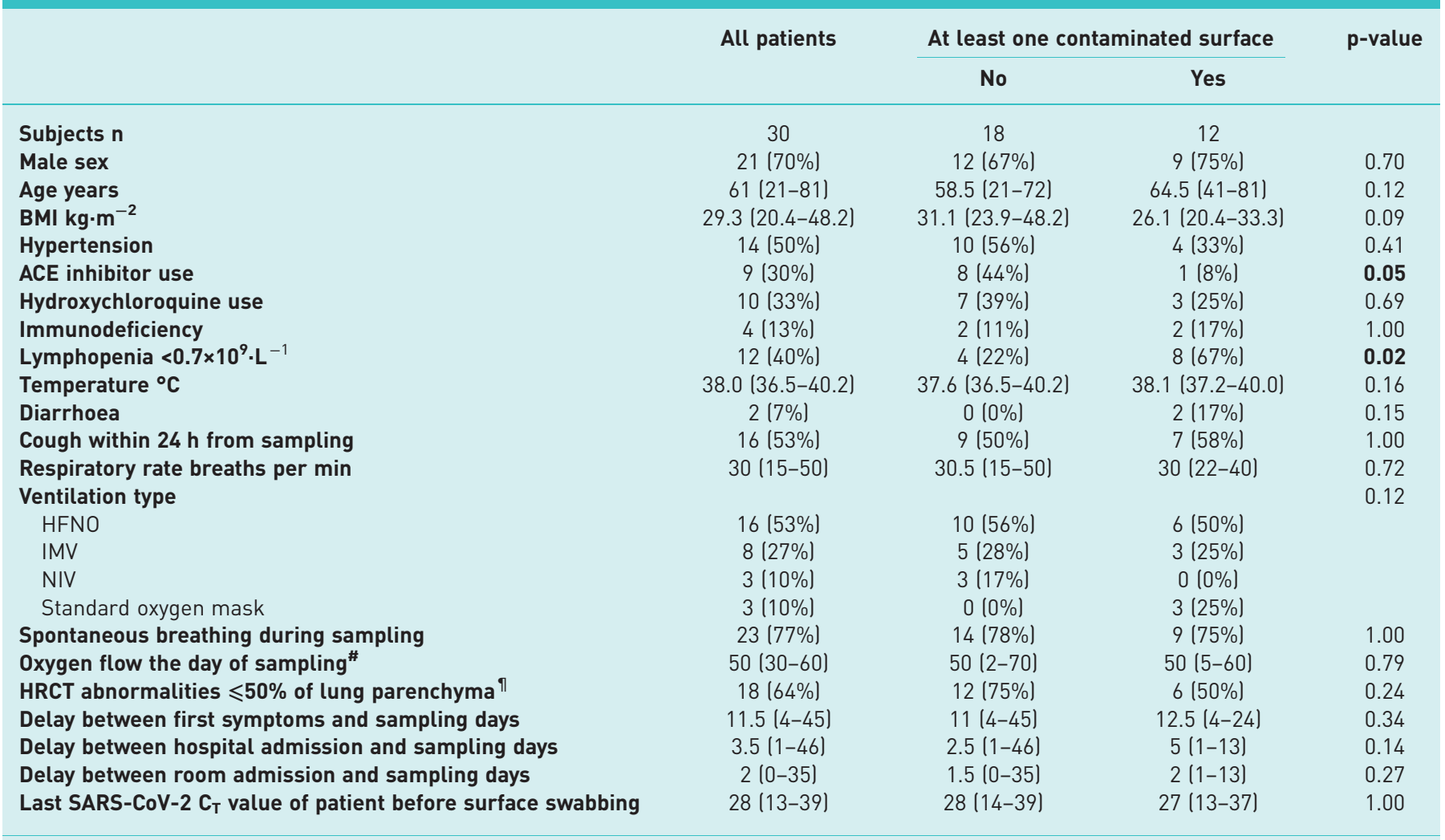

Data are presented as $\mathrm{n}(\%)$ or median (range), unless otherwise stated. BMI: body mass index; ACE: angiotensin converting enzyme; HFNO: high-flow nasal cannula oxygenation; IMV: invasive mechanical ventilation; NIV: noninvasive ventilation; HRCT: high-resolution computed tomography; SARS-CoV-2: severe acute respiratory syndrome coronavirus 2 . ${ }^{\#}: n=21 ;{ }^{~}$ : $n=28$. Bold text indicates $p \leqslant 0.05$.

As all patients were admitted to the ICU, all had severe infection, but none of the usual risk factors for viral spreading were associated with the risk of environmental contamination. Indeed, respiratory symptoms, thoracic HRCT and threshold for patients' real-time PCR positivity were not associated with the risk of environmental contamination. More importantly, despite a low number of events, HFNO does not seem to be associated with a higher risk of contamination. As HFNO is described as an AGP [7], a higher rate of environmental contamination could have been expected. Our results are consistent with LI et al.'s [9] proposal for a rational use of HFNO in COVID-19 patients regarding the risk of environmental contamination.

By univariate analysis, lymphopenia $<0.7 \times 10^{9} \cdot \mathrm{L}^{-1}$ was associated with a higher risk of environmental contamination. Four patients had comorbidity or were on treatment that had an impact on the lymphocyte count, but only two of them had lymphopenia $<0.7 \times 10^{9} \cdot \mathrm{L}^{-1}$. As lymphopenia is common during viral infection, we choose a threshold of $<0.7 \times 10^{9} \cdot \mathrm{L}^{-1}$ as a lymphocyte count below this is uncommon during respiratory virus infections. We hypothesised that the intensity of lymphopenia could highlight an immunological stunning effect due to intense viral invasion. These results should be confirmed in other studies but are of interest as lymphocyte count data are available worldwide.

TABLE 2 Environmental swab results

\begin{tabular}{lccc} 
& Samples $\mathbf{n}$ & Positive $\mathbf{n}(\mathbf{\%}, \mathbf{9 5} \% \mathbf{C I})$ & $\mathbf{C}_{\mathbf{T}}$ median (range) \\
\hline Ventilator control screen & 28 & $8(29,13-49)$ & $35.5(31-38)$ \\
Syringe pump control button & 30 & $7(23,10-42)$ & $37(35-38)$ \\
Bed rails & 30 & $7(23,10-42)$ & $35(29-38)$ \\
Computer table, keyboard and mouse & 29 & $7(24,10-44)$ & $36(34-39)$ \\
At least one positive sample & 30 & $12(40,23-59)$ & $36(29-39)$
\end{tabular}


Since angiotensin-converting enzyme 2 is the receptor for SARS-CoV-2 in human cells, we paid special attention to patients with long-term prescription of ACE inhibitors. We highlighted a statistically significant association by univariate analysis between ACE inhibitors and less environmental contamination that we could not explain, and this needs to be confirmed by further investigation.

Numerous studies have been conducted during the COVID-19 epidemic and showed conflicting results concerning the intensity and modality of SARS-CoV-2 environmental contamination $[3,4,11-18]$. These differences can be explained by several factors. First, some authors did not take into account the patients' profiles in their studies $[4,11,12]$, neglecting the fact that viral shedding in the environment depends not only on viral load but also on the intensity of symptoms [5], time elapsed between the first symptoms and sampling [5, 19], and different types of ventilation [7]. Second, several authors carried out sampling under air treatment conditions that may have affected interpretation of the results [11]. Indeed, the creation of a turbulent flow $[11,20]$ and the negative pressure applied in some studies $[4,12]$ could have modified the risk of environmental contamination. It should be noted that our unit had no air treatment and was under neutral pressure throughout the study period.

One of the strengths of our study lies in taking into account different confounding factors such as the clinical, radiological, therapeutic and virological factors that could have explained or at least influenced the environmental contamination. Also, unlike the other studies, we took the precaution to perform the sampling at least $8 \mathrm{~h}$ after any bio-cleaning to avoid false negative results.

Our results suggest, without being able to demonstrate, that environmental contamination is more frequently linked to indirect contamination than airborne contamination as suggested by some authors [21-25]. In line with previous work, it allows us to reassure healthcare workers of the low risk associated with HFNO [9] and AGP [8].

This study had several limitations. First, it is a monocentric study conducted in an ICU ward without any air treatment. Additionally, the low number of patients and events made a multivariate analysis impossible, thus our exploratory results on lymphopenia and ACE use need to be confirmed by further studies. Furthermore, the presence of SARS-CoV-2 nucleic acids does not necessarily provide information about the infectivity of SARS-CoV-2. As we did not carry out viral culture, viability of the virus is uncertain. Most importantly, this study does not analyse room air or healthcare worker PPE samples. These data could be of importance in healthcare worker contamination. Further studies are needed to confirm our results and to assess airborne contamination.

AIRMIC study group: Jean-Ralph Zahar, Etienne Carbonnelle, Yacine Tandjaoui-Lambiotte, Frédéric Le Gal (Clinical Microbiology Dept, Hôpitaux Universitaires Paris-Seine-Saint-Denis, Bobigny, France), Frédéric Mechai (Infectious and Tropical Diseases Dept, Hôpitaux Universitaires Paris-Seine-Saint-Denis, Bobigny, France), Typhaine Billard-Pomares (Clinical Microbiology Dept, Hôpitaux Universitaires Paris-Seine-Saint-Denis, Bobigny, France), Alexandra Lomont, Chakib Alloui, Nerville-Emmanuel Gordien (Clinical Microbiology Dept, Hôpitaux Universitaires Paris-Seine-Saint-Denis, Bobigny, France), Olivier Bouchaud, Johann Cailhol (Clinical Microbiology Dept, Hôpitaux Universitaires Paris-Seine-Saint-Denis, Bobigny, France), Sophie Brun (Parasitology department, Hôpitaux Universitaires Paris-Seine-Saint-Denis, Bobigny, France), Athenaïs Gerber and Paul Deny (both Clinical Microbiology Dept, Hôpitaux Universitaires Paris-Seine-Saint-Denis, Bobigny, France).

Conflict of interest: None declared.

\section{References}

1 Liu Y, Ning Z, Chen Y, et al. Aerodynamic analysis of SARS-CoV-2 in two Wuhan hospitals. Nature 2020; 582: $557-560$

2 Wilson NM, Norton A, Young FP, et al. Airborne transmission of severe acute respiratory syndrome coronavirus-2 to healthcare workers: a narrative review. Anaesthesia 2020; 75: 1086-1095.

3 Colaneri M, Seminari E, Piralla A, et al. Lack of SARS-CoV-2 RNA environmental contamination in a tertiary referral hospital for infectious diseases in Northern Italy. J Hosp Infect 2020; 105: 474-476.

4 Santarpia JL, Rivera DN, Herrera V, et al. Aerosol and surface contamination of SARS-CoV-2 observed in quarantine and isolation care. Sci Rep 2020; 10: 12732.

5 Huang Y, Chen S, Yang Z, et al. SARS-CoV-2 viral load in clinical samples from critically ill patients. Am J Respir Crit Care Med 2020; 201: 1435-1438.

6 Simonds AK, Hanak A, Chatwin M, et al. Evaluation of droplet dispersion during non-invasive ventilation, oxygen therapy, nebuliser treatment and chest physiotherapy in clinical practice: implications for management of pandemic influenza and other airborne infections. Health Technol Assess 2010; 14: 131-172.

7 Ferioli M, Cisternino C, Leo V, et al. Protecting healthcare workers from SARS-CoV-2 infection: practical indications. Eur Respir Rev 2020; 29: 200068.

8 Tran K, Cimon K, Severn M, et al. Aerosol generating procedures and risk of transmission of acute respiratory infections to healthcare workers: a systematic review. PLoS One 2012; 7: e35797.

9 Li J, Fink JB, Ehrmann S. High-flow nasal cannula for COVID-19 patients: low risk of bio-aerosol dispersion. Eur Respir J 2020; 55: 2000892. 
10 Corman V M, Landt O, Kaiser M, et al. Detection of 2019 novel coronavirus (2019-nCoV) by real-time RT-PCR Euro Surveill 2020; 25: pii $=2000045$.

11 Guo Z-D, Wang Z-Y, Zhang S-F, et al. Aerosol and surface distribution of severe acute respiratory syndrome coronavirus 2 in hospital wards, Wuhan, China, 2020. Emerging Infect Dis 2020; 26: 1583-1591.

12 Faridi S, Niazi S, Sadeghi K, et al. A field indoor air measurement of SARS-CoV-2 in the patient rooms of the largest hospital in Iran. Sci Total Environ 2020; 725: 138401.

13 Ong SWX, Tan YK, Sutjipto S, et al. Absence of contamination of personal protective equipment (PPE) by severe acute respiratory syndrome coronavirus 2 (SARS-CoV-2). Infect Control Hosp Epidemiol 2020; 41: 614-616.

14 Ong SWX, Tan YK, Chia PY, et al. Air, surface environmental, and personal protective equipment contamination by severe acute respiratory syndrome coronavirus 2 (SARS-CoV-2) from a symptomatic patient. JAMA 2020; 323 1610 .

15 Hassanian-Moghaddam H, Zamani N, Kolahi A-A. COVID-19 pandemic, healthcare providers' contamination and death: an international view. Critical Care 2020; 24: 208.

16 van Doremalen N, Bushmaker T, Morris DH, et al. Aerosol and surface stability of SARS-CoV-2 as compared with SARS-CoV-1. N Engl J Med 2020; 382: 1564-1567.

17 Ye G, Lin H, Chen S, et al. Environmental contamination of SARS-CoV-2 in healthcare premises. J Infect 2020; 81: e1-e5.

18 Wang J, Feng $\mathrm{H}$, Zhang S, et al. SARS-CoV-2 RNA detection of hospital isolation wards hygiene monitoring during the Coronavirus Disease 2019 outbreak in a Chinese hospital. Int J Infect Dis 2020; 94: 103-106.

19 Zou L, Ruan F, Huang M, et al. SARS-CoV-2 viral load in upper respiratory specimens of infected patients. N Engl J Med 2020; 382: 1177-1179.

20 Bourouiba L. Turbulent gas clouds and respiratory pathogen emissions: potential implications for reducing transmission of COVID-19. JAMA 2020; 323: 1837-1838.

21 National Academies of Sciences, Engineering, and Medicine. Rapid Expert Consultation on the Possibility of Bioaerosol Spread of SARS-CoV-2 for the COVID-19 Pandemic (April 1, 2020). Washington, DC, National Academies Press, 2020. doi:10.17226/25769.

22 Anderson EL, Turnham P, Griffin JR, et al. Consideration of the aerosol transmission for COVID-19 and public health. Risk Anal 2020; 40: 902-907.

23 Morawska L, Cao J. Airborne transmission of SARS-CoV-2: the world should face the reality. Environ Int 2020; 139: 105730 .

24 Zhao B, Liu Y, Chen C. Air purifiers: a supplementary measure to remove airborne SARS-CoV-2. Build Environ 2020; 177: 106918.

25 Banik RK, Ulrich AK. Evidence of short-range aerosol transmission of SARS-CoV-2 and call for universal airborne precautions for anesthesiologists during the COVID-19 pandemic. Anesth Analg 2020; 131: e102-e104. 